\section{La Etnoeducación}

\section{Afro "Casa Adentro":}

Un modelo

político-pedagógico

en el Pacifico

Colombiano

The Afro ethno-education

"Inside house": A political-

pedagogic model in the

Colombian Pacific

Casa Adentro: Um modelo

político-pedagógico

de etnoeducacão em

afrodescendentes no Pacífico

colombiano

\section{Resumen}

El artículo da cuenta de una propuesta político-pedagógica denominada "Casa Adentro" cuyos cimientos se basan en una pedagogía decolonial diaspórica, que reconoce críticamente la historia de estas comunidades afrodescendientes y recupera los conceptos de ancestralidad y de territorio como fuentes para una educación auténtica. Esta propuesta etnoeducativa traspasa las aulas escolares para convertirse en un escenario pedagógico que vincula a dirigentes comunitarios, padres de familia, promotores culturales y todos los agentes de gestión social y comunitaria que impulsan el crecimiento de las sociedades afro en el Pacifico.

\section{Palabras clave}

Casa Adentro, etnoeducación-afro, pedagogía decolonial, ancestralidad, territorio, sistermatización de experiencia.

\section{Abstract}

This article deals with a political-pedagogical proposal called "Inside House" which foundations are based on a diaspora de-colonial pedagogy, which recognizes critically the history of these afro-descendant communities and recuperates the concepts of ancestral and territory as sources for a real education. This ethno-educational proposal goes beyond the classrooms, in order to become in a second pedagogic scenery, which links to community leaders, parents, cultural developers, and all of the social, community manager agents, who promote the growing of afro societies in the pacific.

\section{Key words}

Inside house, afro ethno-education, de-colonial pedagogy, ancestral, territory, systematization of experiences.

\section{Resumo}

0 artigo apresenta uma proposta político-pedagógica chamada Casa Adentro, baseada em uma pedagogia da diáspora descolonizadora, que reconhece uma história crítica dessas comunidades afrodescendentes e recupera os conceitos de ancestralidade e de território como fontes da educação autêntica. Esta proposta vai além da sala de aula e transforma-se no contexto pedagógico que integra líderes comunitários, pais, promotores culturais e agentes de gestão social e comunitária que estimulam o crescimento das sociedades afro no Pacífico.

\section{Palavras-chave}

Casa Adentro, etnoeducação afro, pedagogia descolonizadora, território, ancestralidade, sistematização de experiência.

Fecha de recepción: 11 de marzo de 2011 Fecha de aprobación: 22 de abril de 2011

Pedagogía y Saberes No. 34 Universidad Pedagógica Nacional Facultad de Educación. 2011, pp. 117 - 121 
$\mathrm{D}$ esde la década de los años ochenta, algunos sectores organizados de las comunidades afrodescendientes en Colombia están planteando y construyendo propuestas de educación en el marco de sus tradiciones culturales (Escuela la Playa Salahonda, Nariño), de sus intereses organizativos (Villa Paz Jamundí, Valle) y en la recuperación de sus lenguas vernáculas (San Basilio de Palenque, Bolívar, y San Andrés Islas). Posteriormente, al principio de la década de los años noventa, el Ministerio de Educación Nacional declaró estas experiencias como proyectos etnoeducativos ${ }^{1}$. Por esa época se asistió a la construcción de lo que podría llamarse una agenda política de las comunidades negras en Colombia.

Como se sabe, las discusiones al interior de las comunidades generadas por el artículo 55 transitorio de la Constitución dio como resultado la ley 70 de 1993. Los temas allí tratados y posteriormente convertidos en norma tienen un gran nivel de importancia entre los afrocolombianos; sin embargo, dados los extenuantes debates suscitados en el comienzo de este proceso y la profundidad con la cual se abordaron se podría decir, casi sin equivocación, que los dos pilares de la agenda afro en los años noventa fueron la etnoeducación y la territorialidad. Razón por la cual, en especial en el Pacifico, se asocia etnoeducación con defensa del territorio y funge, de esta manera, una visión político-educativa que acompañará los debates acerca de la pertinencia de los procesos de formación en el ámbito de los pueblos, veredas y regiones afrocolombianas.

En los primeros diez años del siglo XXI los debates han girado en torno a la necesidad de construcción de Sistemas Educativos Propios². Al parecer, la noción de etnoeducación ya se trona insuficiente para nombrar lo que de fondo se desea: una educación diseñada, gestionada y realizada desde las comunidades en un escenario propio con una concepción "Casa adentro" (Cfr, García, 2000). Y no es para menos, la etnoeducación pasó de ser una propuesta alternativa de los pueblos para la realización de sus planes de vida y proyectos de etnodesarrollo a convertirse en parte del discurso oficial. En efecto, detrás del concepto de etnoeducación no solo existen percepciones distintas sino contrarias. No se considera una invención asegurar que la cuestión etnoeducativa en el discurso oficial esconde la idea de una educación devaluada y marginal. Por otro lado, no es desconocido que la construcción de una etnoeducación como modelo

1 Primer Seminario Taller de Etnoeducación Afrocolombiana. Memorias. Ministerio de Educación. 1994.

2 Talleres de Etnoeducación en la Costa de Nariño, apoyados por el Fondo Mundial para la Naturaleza WWF. Tumaco 2004. pedagógico propio en el Pacifico se constituye en la apuesta de la comunidad afro por un proyecto de reconstrucción de la identidad en términos políticos y por la autodeterminación.

Ahora bien, el concepto "Casa adentro" no solo lleva implícita una categoría territorial: se reconoce también, y quizás más profundamente, como un escenario de construcción de pensamiento propio que centra los debates acerca del deber ser de la educación en lo más íntimo, en lo que atañe a la ancestralidad, pero sobre todo en lo políticamente conveniente. En otras palabras, la educación "casa adentro" se aleja de los discursos estandarizados promovidos por los gobiernos y asume la construcción de una propuesta educativa de acuerdo con parámetros relacionados con la visión autonómica de los pueblos.

\section{¿Cómo se concibe conceptualmente el modelo desde el Pacifico Sur?}

Si bien la etnoeducación se considera una corriente o tendencia pedagógica que abarca un número indeterminado de propuestas educativas que responden a la diversidad de enfoques, posturas políticas y prácticas culturales de los afrocolombianos, el Pacifico ha adoptado hace ya un buen tiempo la construcción de un modelo pedagógico en donde el territorio se constituye en elemento esencial. Es más, en la medida en que en el Pacifico la línea que divide lo urbano de lo rural es realmente difusa, poco a poco el territorio se va convirtiendo en "región" o en Comarca como lo han señalado los hermanos afroecuatorianos ( $\mathrm{Cfr}$. García, 2001).

Este hecho se torna definitivo para sumar políticamente en cuanto ya no solo se consideran ancestrales los territorios negros colectivos, apartados y confinados por efectos de la guerra, sino que estas nociones gravitan en forma permanente en el discurso pedagógico de las comunidades llámense rurales o urbanas. Ahora bien, el territorio, región o comarca se percibe como propiedad colectiva, como legado ancestral, como lugar de autonomía y espacio de libertad. No fueron en vano los ejercicios de re-lectura de la historia que se realizan desde el año $1991^{3}$. Lo cual aparece más claro en estos debates es que la población afro de los territorios del Pacifico vienen de una historia de esclavización que no solo no ha terminado sino que se reedita cíclicamente con la presencia de inversionistas externos en procesos

3 Encuentros, talleres, conversatorios del Palenque Regional Nariño previo y posterior a la promulgación de la Constitución Nacional. 
de extracción de los recursos naturales ${ }^{4}$. Si se analiza, entonces, el neocolonialismo en los territorios afrocolombianos, se impone la necesidad de crear condiciones para dar continuidad a los procesos de liberación. Partir de estas reflexiones implica construir una educación cuyos contenidos esenciales se relacionan íntimamente con el proyecto cultural de la comunidad afrocolombiana que no solo apela a los ancestros para restituir prácticas productivas, culinarias o artísticas sino que busca con afán en la voz de la narrativa propia los vestigios de una concepción política del territorio.

De esta manera, en la comunidad negra del $\mathrm{Pa}$ cifico, concretamente en la Costa de Nariño, poco a poco se va develando el sentido profundo de una pedagogía decolonial afrodiaspórica. Esta pedagogía, en proceso de concertación, comporta dos ideas fuertes: por un lado, la re-construcción de un sujeto colectivo con identidad política y, por otra parte, el aspecto metodológico donde todos los saberes se apropian y se comprenden en la cotidianidad de las construcciones culturales propias. De la misma manera, se puede afirmar que en esta región se ha venido aplicando una especie de heurística en las relaciones socio-culturales de la comunidad afropacífica del pasado. Una forma de búsqueda de lo propio, de redescubrimiento de sí mismo. Si bien no existen escritos antiguos, la heurística está referida a la búsqueda del esencialismo afro en la tradición oral y en una resocialización analítica y cualitativa de la memoria ancestral que es capaz de dar testimonio de libertad y vida autónoma en el Pacifico.

Sin embargo, el proyecto cultural afropacifico ha sido invisibilizado en los últimos tiempos a través de la estrategia de la folclorización. Si bien lo artístico se constituye en un aspecto importante de la identidad, no es en sí mismo la representación de la región. Más allá de este aspecto simbólico, aparece una sociedad construida sobre el principio de la solidaridad o del hermanamiento entre los seres humanos y de estos con el territorio. Los cimientos de esta estructura social han sido afectados por la presencia de proyectos externos, legales o ilegales, estatales o extranjeros que han intentado penetrar en lo más intimo de la cultura del Pacifico lesionando severamente las creencias, prácticas de convivencia y hasta la forma

4 En las primeras décadas del siglo XX explotaron la tagua hasta el agotamiento de la especie, luego se pasó al corte del mangle para la extracción del tanino, después vino la presión sobre los recursos maderables y al final del siglo la introducción del monocultivo de la palma aceitera y la devastación de manglares para la cría de camarón en cautiverio. Ninguno de estos proyectos de inversión externa, algunos con el auspicio del gobierno, está exonerado de haber ingresado en la región del Pacifico con estrategias de desarraigo y esclavización. de actuar y de pensar de gran parte de la comunidad afrocolombiana.

La heurística, entonces, consiste en hundirse en las raíces y leer de nuevo en el legado socio-cultural de los primeros habitantes africanos y sus descendientes. Fueron cimarrones, sin duda, quienes se inventaron una sociedad en lo más insondable de la manigua en un acontecimiento que no puede concebirse más que como un acto de autonomía. Es decir, por encima de la concepción antropológica de la territorialidad, lo que sucede en esta Comarca es también una hermenéutica del lenguaje político de la ancestralidad. Aquí las preguntas giran en torno a cuáles fueron los códigos que instauraron los mayores para el establecimiento de comunidades autodeterminadas y con capacidad de defensa del espacio de vida. Estos interrogantes y otros que en materia de relación con la naturaleza se formulan, se hacen de manera recurrente en el proceso de replanteamiento del modelo educativo en el Pacifico. Dichos interrogantes tienen el carácter de preguntas de análisis e interpretación del pensamiento de los forjadores de la Sociedad Afropacifica y buscan respuestas que actualicen el contenido político de ese pensamiento. En efecto, la narrativa del Pacifico abunda en frases, dichos, refranes, coplas, decimas, cuentos y leyendas, cuya correcta interpretación dará la clave para develar no solo el concepto de sociedad existente, también los procedimientos para reconstruirla a luz de las realidades actuales.

Por otro lado, la etnoeducación "casa adentro" no es un modelo para ser aplicado exclusivamente en el sector educativo formal. Se trata también de una estrategia y de un escenario de aprendizaje para dirigentes comunitarios, padres de familia, promotores culturales y todos los agentes de gestión social y comunitaria que impulsan el crecimiento de las sociedades afro en el Pacifico. Desde esta perspectiva, se considera posible hablar de fortalecimiento de los espacios de formación comunitaria tendientes a la cohesión y a la organización de las poblaciones en torno a las problemáticas que les incumben directamente y a la búsqueda endógena de caminos que lleven a la superación de las precarias condiciones materiales de vida, elevando la conciencia social y su pertenencia cultural. Estos presupuestos inducen a crear espacios permanentes de reflexión al interior de las comunidades para lograr mayor solidez y coherencia en sus concepciones políticas. Los programas de etnoalfabetización con jóvenes y adultos ${ }^{5}$,

5 Hace referencia a los programas de Etnoalfabetización y Etnobachillerato que se desarrollan en Recompas (Red de Consejos Comunitarios del Pacifico Sur). Estos programas tienen el apoyo del Consejo Noruego para Refugiados. 
los encuentros de saberes ancestrales, los procesos de formación política a los dirigentes, los eventos de construcción de planes de etnodesarrollo, y la formulación colectiva de reglamentos internos de convivencia ${ }^{6}$, entre otros, son algunos de los signos inequívocos de la etnoeducación "casa adentro" más allá de las aulas escolares.

No obstante, se considera pertinente reconocer que la propuesta etnoeducativa formal, al plantearse una articulación entre escuela y comunidad, responde también al interés político de cohesión comunitaria y profundiza en el concepto de liderazgo en el ámbito de la acción de los maestros etnoeducadores.

\section{¿Cómo se viene construyendo el modelo en la comunidad Afropacifica?}

Los cuestionamientos a los programas y a las orientaciones generales que ofrecen las autoridades educativas oficiales, las jornadas de re-pensamiento al interior de los consejos comunitarios y demás organizaciones étnico-territoriales, los materiales pedagógicos elaborados, las capacitaciones con los docentes, los encuentros de cantaoras, decimeros, curanderos y cultores de otros saberes ancestrales, la construcción de currículo propio y en fin la infinidad de espacios de reflexión habilitados se reconocen como algunos indicadores ciertos de que las comunidades afro del Pacifico vienen en una construcción permanente de un modelo pedagógico con intereses de autenticidad.

Este proceso de diseño teórico-práctico de una educación propia en la comunidad negra cuenta con un elemento esencial que lo hace diferente de otros modelos. No es resultado de elucubraciones u observaciones científicas de un autor especifico; se constituye, ante todo, en un acumulado de sentimientos, de aportes académicos, de intercambios pedagógicos, de reconocimiento de las pautas de crianza, análisis de los modos de transmisión de los saberes desde los mayores, análisis político de la territorialidad, reflexiones sobre la colonialidad de la historia común afrodescendiente en Colombia y ejercicios de reafirmación de la identidad, entre otros aspectos, que han sido realizados por las distintas organizaciones que integran el movimiento social afrocolombiano especialmente en su expresión afropacífica.

6 Se refiere a los Planes de Etnodesarrollo y Reglamentos Internos de gobernabilidad que vienen construyendo los 14 consejos comunitarios que integran Recompas.
Es decir, se trata de una construcción colectiva de la misma comunidad y no de la obra de autores monumentales como Piaget, Vigotsky, Bruner, Montessori o Pestalozzi. En efecto, los maestros etnoeducadores, los líderes de los consejos comunitarios, las comisiones pedagógicas creadas alrededor de la ley 70 de 1993, la Pastoral Afro, los jóvenes de las universidades, las agrupaciones artísticas, los grupos de mayores conocedores de saberes ancestrales han aportado cada uno en su nivel, en su espacio y en su momento variados elementos de corte religiosoespiritual, ambiental, económico, social y político que han ido delineando el proyecto educativo propio de la comunidad afro en el Pacifico.

Previo al diseño de un esquema que represente el modelo etnoeducativo en el Pacifico, se crean escenarios de debate en relación con el contexto regional, tomando como base un análisis profundo del sistema colonial que definió un patrón de poder enderezado a subalternizar a grupos humanos por su condición racial (Cfr. Quijano, 2000). En efecto, el fenómeno del colonialismo desarrollado por los europeos en América desde el siglo XV y ahora, en los tiempos contemporáneos, traducido a nuevas estrategias de esclavización es el punto de partida para esbozar la construcción de los pilares de una sociedad con un modelo de educación anti-hegemónico. Pero un diagnostico completo de la situación de los negros del Pacifico pasa también por un autoexamen de sus potenciales como pueblo y de los recursos que posee su territorio. Por ello, se retoma también el afianzamiento de valores claves para la convivencia social.

Valores como el de la solidaridad que ha significado el punto articulador de las sociedades del Pacifico y las construcciones culturales que lo promueven marcan la pauta en la definición del horizonte de estas comunidades. Con estas reflexiones, no solo se facilita la identificación de acciones para mantener los potenciales comunitarios, también se va penetrando en la conciencia de los pueblos negros del Pacifico el carácter liberador del proyecto educativo que se busca.

En suma, "casa adentro" consiste en la estrategia elegida por los animadores y constructores de los procesos educativos propios en cumplimiento del mandato ancestral de los forjadores de la cultura y el pensamiento afro en el Pacifico. "Casa adentro", como concepto, alude a la construcción de sociedad soportada en un interés colectivo con un claro propósito de diferenciación, apropiación y defensa de unos valores, creencias, pensamientos y principios de relacionamiento con el entorno; se asocia, entonces, con la autonomía de las comunidades en la toma decisiones al 
interior de sus territorios pero también con el rechazo a una imposición desde afuera a la hora de definir los pilares que guiaran su proyecto de sociedad.

En el campo etnoeducativo, se construye "casa adentro" cuando los componentes del proyecto se discuten y se acuerdan por los miembros de la comunidad, y solo por estos, en un ejercicio de autodeterminación. Esta es la tendencia en los procesos

\section{Bibliografía}

García, S. J. (2001), Cuentos de la Creación. Comarca Territorial del Norte de Esmeraldas, FEDARPOM.

, La Tradición Oral, una herramienta para la etnoeducación, Quito, Ecuador. de construcción de educación propia en el Pacifico Sur (Costa de Nariño) que reconoce la necesidad de acudir en forma permanente a la voz de los mayores en un acto de interpretación de su visión de mundo (hermenéutica del pensamiento político ancestral) y en un penetrar en las honduras de sus tradiciones y prácticas de vida (heurística de las relaciones sociales y culturales de la comunidad afro del Pacifico).

Quijano, A., (2000), Colonialidad del Poder, eurocentrismo y América Latina, Buenos Aires, Argentina. 


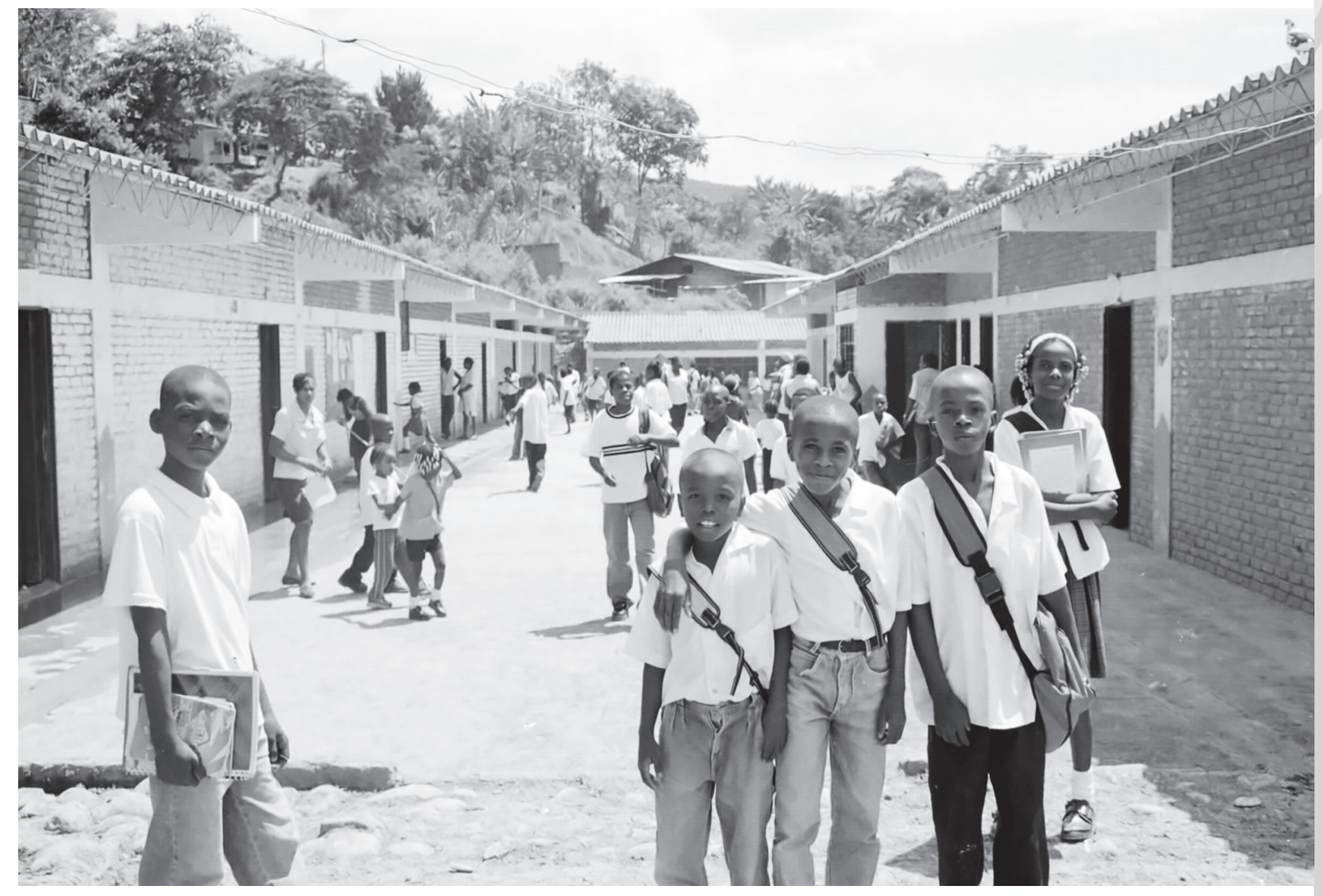

Fotografía. Centro de Memorias Étnicas de la Universidad del Cauca 